\title{
A pair of close YSOs with strikingly different outflow ejection geometry
}

José M. Torrelles ${ }^{1}$, Nimesh A. Patel $^{2}$, Guillem Anglada ${ }^{3}$, José F. Gómez $^{4}$, Paul T. P. Ho ${ }^{2}$, Lucas Lara ${ }^{5}$, Antonio Alberdi ${ }^{3}$, Jorge Cantó $^{6}$, Salvador Curiel ${ }^{6}$, Guido Garay ${ }^{7}$ and Luis F. Rodríguez ${ }^{8}$

${ }^{1}$ Instituto de Ciencias del Espacio (CSIC) and Institut d'Estudis Espacials de Catalunya, Gran Capità, 2-4, 08034 Barcelona, Spain. On sabbatical leave at the UK Astronomy Technology Centre, Royal Observatory Edinburgh

${ }^{2}$ Harvard-Smithsonian Center for Astrophysics, 60 Garden Street, Cambridge, MA 02138, USA

${ }^{3}$ Instituto de Astrofísica de Andalucía (CSIC), Apdo. 3004, 18080 Granada, Spain

${ }^{4}$ Laboratorio de Astrofísica Espacial y Física Fundamental (INTA), Ap. 50727, 28080 Madrid, Spain

${ }^{5}$ Departamento de Física Teórica y del Cosmos, Facultad de Ciencias, Universidad de Granada, 18071 Granada, Spain

${ }^{6}$ Instituto de Astronomía (UNAM), Ap. 70-264, DF 04510, México

${ }^{7}$ Departamento de Astronomía, Universidad de Chile, Casilla 36-D, Santiago, Chile

${ }^{8}$ Centro de Radioastronomía y Astrofísica (UNAM), Ap. 72-3, 58089 Morelia, Michoacán, México

\begin{abstract}
We present Very Long Baseline Array (VLBA) proper motion observations of water masers toward two young stellar objects (YSOs) of the W75N(B) high-mass star forming region. These observations (Torrelles et al. 2003) show two objects having a similar spectral type (early-B stars), separated in the sky by 0.'7 (corresponding to $1400 \mathrm{AU}$ at the source distance), sharing the same molecular gas environment, but presenting a strikingly different outflow ejection geometry. One of these YSOs, W75N(B)-VLA 1, has a jet-like outflow at 2000 AU scale, with the water masers moving at velocities of $\simeq 20 \mathrm{~km} \mathrm{~s}^{-1}$ along the major axis of the thermal radio continuum jet, while the other YSO has a water maser shell outflow of 160 AU radius expanding at $\simeq 30 \mathrm{~km} \mathrm{~s}^{-1}$ in multiple directions with respect to the central compact radio continuum source W75N(B)-VLA 2. Given the small dynamical time obtained for the water maser shell ( $\simeq 13 \mathrm{yr}$ ), we propose that in the very early stages of the star-formation process there may exist short lived, possibly repetitive, events associated with very poorly collimated outflows. All these results suggest that outflow collimation is not only a consequence of ambient conditions, but something intrinsic to the evolution of the individual stars, opening the important question of how and when these non-collimated wind ejection events occur in the evolution of YSOs. Although we argue in this paper that W75N(B)-VLA 2 could be in an earlier stage of evolution than W75N(B)-VLA 1, we think that observations at (sub)millimeter wavelengths with angular resolutions of $\simeq 0$.' 1 can provide the key for determining the relative stage of evolution of these two remarkable YSOs.
\end{abstract}

Keywords. ISM: jets and outflows-masers-stars: formation

\section{Introduction}

W75N is a high-mass star forming region located at a distance of $2 \mathrm{kpc}$ (Dickel, Wendker, \& Bieritz 1979, Wynn-Williams, Becklin, \& Neugebauer 1974), in the Cygnus X complex, with an integrated IRAS luminosity of a few times $10^{5} L_{\odot}$ (Moore et al. 1988). It contains a subregion, W75N(B), which comprises a large-scale CO outflow extending 
over several parsecs, a cluster of ultracompact HII regions, several millimeter and infrared continuum sources, and strong $\mathrm{H}_{2} \mathrm{O}$ and $\mathrm{OH}$ maser emission (Haschick et al. 1981, Fisher et al. 1985, Hunter et al. 1994, Davis et al. 1998, Torrelles et al. 1997, Shepherd 2001, Hutawarakorn, Cohen, \& Brebner 2002, Slysh et al. 2002, Shepherd, Testi, \& Stark 2003, Niezurawaska et al. 2004, Shepherd, Kurtz, \& Testi 2004, Persi, Tapia, \& Smith 2005). At much smaller scales $\left(\simeq 1^{\prime \prime}, \simeq 2000 \mathrm{AU}\right)$, towards the center of the extended molecular outflow, there are three compact radio continuum sources which were detected with the Very Large Array (VLA), VLA 1[W75N(Ba)], VLA 2, and VLA 3[W75N(Bb)], which are probably excited by early-B type stars, and two clusters of water masers, one associated with VLA 1, and the other one with VLA 2 (Torrelles et al. 1997) (two of the compact radio continuum sources, W75N(Ba) and $\mathrm{W} 75 \mathrm{~N}(\mathrm{Bb})$, were first detected by Hunter et al. 1994). The masers associated with VLA 1 are distributed along the major axis of the observed elongated radio continuum emission at scales of $\simeq 1^{\prime \prime}$. This fact, together with the spectral index $\alpha_{(3.6-1.3 \mathrm{~cm})}=0.7 \pm 0.1\left(\mathrm{~S}_{\nu} \propto \nu^{\alpha}\right)$ obtained for VLA 1 (which is consistent with an ionized thermal jet), suggests that VLA 1 could be a radio jet in which the water masers are tracing the base of a collimated outflow. On the other hand,

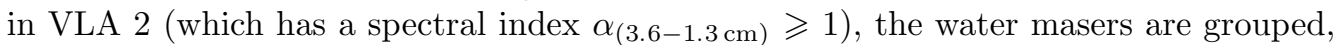
forming a shell of $\simeq 0$ " 1 radius $(\simeq 200$ AU) around the compact continuum source, and it was suggested that these masers could trace bound motions in a circumstellar disk (Torrelles et al. 1997). VLA 1 and VLA 2 are separated in the sky by $\simeq 00^{\prime \prime} 7(\simeq 1400$ AU), without any counterpart at mid-infrared wavelengths (Persi, Tapia, \& Smith 2005).

We have tested the scenarios proposed for VLA 1 and VLA 2 by measuring proper motions of the associated water masers through VLBA (NRAO†) multi-epoch observations. We used the VLBA because of its high ability to track proper motions of masers on a much shorter time baseline (a few weeks) and spatial resolution $(\simeq 0.5$ milliarcseconds, $\simeq 1 \mathrm{AU}$ at the source distance), compared to the VLA. The results of the VLBA water maser observations have been already presented and extensively discussed in Torrelles et al. (2003). In this paper we present (§ 2) a summary of the main VLBA results. In addition, we also discuss $(\S 3)$ on the relative stage of evolution of VLA 1 and VLA 2.

\section{Water maser proper motions toward VLA 1 and VLA 2: jet-like (VLA 1) and shell-like (VLA 2) outflows}

The VLBA observations have shown that while the proper motions of the water masers in VLA 1 are parallel to the thermal radio jet, in VLA 2 the masers are distributed in a shell with their proper motions moving outwards in multiple directions from the central radio continuum source (Torrelles et al. 2003).

In particular, the VLBA water masers around VLA 1 are distributed on a linear structure of $\simeq 2000$ AU size (with a small spatial dispersion in width of $200 \mathrm{AU}$ ), having proper motions of $\simeq 2$ milliarcseconds (mas) per year $\left(\simeq 19 \mathrm{~km} \mathrm{~s}^{-1}\right)$ parallel to the thermal radio jet, a mean line-of-sight velocity $V_{\mathrm{LSR}} \simeq 12 \mathrm{~km} \mathrm{~s}^{-1}$ (close to the systemic velocity of the $\mathrm{W} 75 \mathrm{~N}$ cloud $\left(V_{\mathrm{LSR}} \simeq 10 \mathrm{~km} \mathrm{~s}^{-1}\right)$, and a very small LSR velocity dispersion of $\simeq 1.5 \mathrm{~km} \mathrm{~s}^{-1}$. These results imply that the water masers in VLA 1 are tracing a highly collimated outflow with an opening angle of $\simeq 10^{\circ}$ and moving almost on the plane of the sky $\left(i \geqslant 80^{\circ}\right)$, supporting the previous interpretation based on VLA observations (Torrelles et al. 1997).

$\dagger$ The National Radio Astronomy Observatory is a facility of the National Science Foundation operated under cooperative agreement by Associated Universities, Inc. 
On the other hand, the masers associated with VLA 2 are distributed on a shell of $\simeq 160$ AU radius, moving outward from the compact radio continuum source in multiple directions, with proper motions of $\simeq 3$ mas $\mathrm{yr}^{-1}\left(\simeq 28 \mathrm{~km} \mathrm{~s}^{-1}\right)$, having a mean line-ofsight velocity $V_{\mathrm{LSR}} \simeq 5 \mathrm{~km} \mathrm{~s}^{-1}$, and a relatively large LSR velocity dispersion of $\simeq 6 \mathrm{~km}$ $\mathrm{s}^{-1}$. These large velocities would require a binding mass of $\simeq 100 \mathrm{M}_{\odot}$, which is not observed. Therefore, both the magnitude and the direction of the observed velocities imply expanding motions, discarding that the masers trace bound motions in a circumstellar disk as it was suggested by previous VLA observations (Torrelles et al. 1997).

We have considered several scenarios for the driving agent of this expanding water maser shell, but the one most likely appears to be a wind-driven shell with a mass loss rate $\dot{M}_{w} \simeq 8 \times 10^{-7} M_{\odot} \mathrm{yr}^{-1}$ and velocity $V_{w} \simeq 100 \mathrm{~km} \mathrm{~s}^{-1}$. In addition, the estimated age (dynamical time) of the water maser shell is $t \simeq 13 \mathrm{yr}$. Given how unlikely it would be to observe this short-lived phenomena if it happened only once in the life time of the YSO, we suggested that at the beginning of stellar evolution, there may exist important, possibly short lived repetitive events associated with very poorly collimated outflows (Torrelles et al. 2003).

Non-collimated and isotropic outflows have been also reported in other star forming regions (Patel et al. 2000, Shepherd, Claussen, \& Kurtz 2001, Torrelles et al. 2001, Gallimore et al. 2003), which in general are difficult to explain within a scenario of a protostar surrounded by a circumstellar disk, where a preferred direction in the outflow is expected (Bally \& Zinnecker 2005 have proposed that wide-angle outflows could arise as a consequence of protostellar mergers, but we do not find evidence of such event in our very high angular resolution data of $\mathrm{W} 75 \mathrm{~N}(\mathrm{~B}))$. The importance of our results on $\mathrm{W} 75 \mathrm{~N}(\mathrm{~B})$ is the well differentiated outflow geometry found simultaneously in two close (1400 AU) similar (B-early type) YSOs, suggesting that outflow collimation is not only a consequence of ambient conditions (because both objects share the same molecular core environment), but something intrinsic to the individual evolution of stars, and therefore opening the important question of how and when the non-collimated wind ejection stage occurs in the evolution of YSOs.

\section{Differences in the relative stage of evolution of VLA 1 and VLA 2?}

We do not know which YSO (VLA 1 or VLA 2) is at an earlier stage of evolution, and consequently we do not know whether the shell outflow is produced before or after the stage of the jet outflow. However, we argue that since the shell outflow from VLA 2 is observed at a smaller scale (and has a smaller dynamical time) than the collimated motions from VLA 1, VLA 2 could be in an earlier stage than VLA 1. Furthermore, since the spatial dispersion of the water masers from the major axis of the VLA 1 radio jet $(\simeq 200 \mathrm{AU})$ is similar to the scale where the VLA 2 shell outflow is observed, we speculate that the outflow from VLA 2 could evolve in the future into a collimated jet as in VLA 1. If so, it would introduce a variant in our usual belief that the onset of outflow activity, in the earliest stages of YSOs, already occurs with a high degree of collimation.

It is believed that the ratio of submillimeter to bolometric luminosity traces the mass of the envelope that is being accreted into the star (André et al. 2000). Then, it is expected that this ratio is higher in the earliest stages and decreases with time. Therefore, we think that observations at (sub)millimeter wavelengths with angular resolutions of $\simeq 0$.' 1 are the key studies for determining the relative stage of evolution of these two remarkable YSOs in $\mathrm{W} 75 \mathrm{~N}(\mathrm{~B})$. 


\section{Acknowledgements}

AA and LL are supported by MECD grant AYA2001-2147-CO2. GA, JFG, and JMT acknowledge support from MECD grant AYA2002-00376 (including FEDER funds). AA, GA, and LL acknowledge support from Junta de Andalucía.

\section{References}

André, P., Ward-Thompson, D., \& Barsony, M. 2000, in Protostars and Planets IV, eds. V. Mannings, A. P. Boss, S. S. Russell (Tucson: University of Arizona Press), p. 59

Bally, J. \& Zinnecker, H. 2005, AJ 129, 2281

Davis, C. J., Moriarty-Schieven, G., Eislöffel, J., Hoare, M. G., \& Ray, T. P., 1998, AJ 115, 1118.

Dickel, H. R., Wendker, H. J., \& Bieritz, J. H. 1969, A\&A 1, 270

Fischer, J., Sanders, D. B., Simon, M., \& Solomon, P. M., 1985, ApJ 293, 508

Gallimore, J. F., Cool, R. J., Thornley, M. D., \& McMullin, J. 2003, ApJ 586, 306

Haschick, A. D., Reid, M. J., Burke, B. F., Moran, J. M., \& Miller, G. 1981, ApJ 244, 76

Hunter, T. R., Taylor, G. B., Felli, M., \& Tofani, G., 1994, A\&SA 284, 215

Hutawarakorn, B., Cohen, R. J., \& Brebner, G. C., 2002, MNRAS 330, 349

Moore, T. J. T., Mountain, C. M., Yamashita, T., \& Selby, M. J. 1988, MNRAS 234, 95

Niezurawaska, A., Szymczak, M., Cohen, R. J., \& Richards, A. M. S. 2004, MNRAS 350, 1409

Patel, N. A., Greenhill, L. J., Herrnstein, J., Zhang, Q., Moran, J. M., Ho, P. T. P., \& Goldsmith, P. F. 2000, ApJ 538, 268

Persi, P., Tapia, M., \& Smith, H. A. 2005, $A \& A$, in press

Shepherd, D. S. 2001, ApJ 546, 345

Shepherd, D. S., Claussen, M. J., \& Kurtz, S. E. 2001, Science 292, 1513

Shepherd, D. S., Kurtz, S. E., \& Testi, L. 2004, ApJ 601, 952

Shepherd, D. S., Testi, L., \& Stark, D. P. 2003, AJ 584, 882

Slysh, V. I., Migenes, V., Val'tts, I. E., Lyubchenko, S. Y., Horiuchi, S., Altunin, V. I., Fomalont, E. B., \& Inoue, M., 2002, ApJ 564, 317

Torrelles, J. M., Gómez, J. F., Rodríguez, L. F., Ho, P. T. P., Curiel, S., \& Vázquez, R., 1997, ApJ 489, 744 .

Torrelles, J. M., Patel, N., Anglada G., Gómez, J. F., Ho, P. T. P., Cantó, J., Curiel, S., Lara, L., Alberdi, A., Garay, G., \& Rodríguez, L. F. 2003, ApJ 598, L115

Torrelles, J. M., Patel, N. A., Gómez, J. F., Ho, P. T. P., Rodríguez, L. F., Anglada, G., Garay, G., Greenhill, L., Curiel, S., \& Cantó, J. 2001, Nature, 411, 277

Wynn-Williams, C. G., Becklin, E. E., \& Neugebauer, G. 1974, ApJ 187, 473 